\title{
La microscopie optique à sonde locale
}

\author{
D. van Labeke \\ Laboratoire d'Optique P.M. Duffieux, Université de Franche-Comté, URA 214 du CNRS, \\ UFR Sciences et Techniques, route de Gray, 25030 Besançon cedex, France
}

\begin{abstract}
Résumé : cet article est le texte d'un cours destiné à présenter la microscopie optique à sonde locale et décrire ses applications. La première partie sert d'introduction, décrit le contexte historique, et explique comment, depuis le début des années 1980, les nouvelles microscopies à sonde locale permettent d'obtenir des images avec une résolution inespérée. Le chapitre suivant décrit et compare les principales configurations de microscopes optiques à sonde locale. Puis nous donnons le principe de fonctionnement de ces microscopes; nous montrons le rôle des ondes évanescentes et expliquons comment on peut dépasser la limite de résolution de Rayleigh tout en respectant les relations d'incertitude. Nous discutons aussi rapidement le problème de la résolution de ces microscopes et l'influence de la distance sonde-objet. Le chapitre suivant aborde les problèmes techniques et leurs solutions. Finalement nous présentons les principaux résultats et décrivons les applications les plus significatives : en spectroscopie, biologie, contrôle optique, stockage de masse, physique du solide.
\end{abstract}

\section{INTRODUCTION ET CONTEXTE}

Le premier microscope a été conçu et réalisé par le Hollandais Van Loeuvenhook vers 1590. Depuis des innovations ont permis des améliorations de l'image ou de son contraste (microscope composé, objectif achromatique, microscope polarisant, condenseur, contraste de phase, contraste interférentiel) mais, pendant presque 4 siècles, jusqu'en 1980 , mis à part des changements de " design " ou d'esthétique, tous les microscopes conservent la même forme et le même principe de fonctionnement. Le microscope électronique, qui date du milieu du $\mathrm{XX}^{\mathbf{e}}$ siècle repose également sur le même principe de fonctionnement, des électrons remplaçant la lumière.

En microscopie optique traditionnelle, l'objet à étudier est éclairé par transmission ou réflexion et la lumière diffractée est captée par une lentille objective, située à grande distance de l'objet et servant à en faire une image.

Le but d'un microscope étant de distinguer des détails, une des qualités fondamentales est le pouvoir de résolution. En fait, il faut distinguer deux types de résolution :

- La résolution dans la direction perpendiculaire au plan de l'objet ne semble pas avoir de limite. Par interférométrie, en observation visuelle, on parvient à déceler des variations de 
hauteur de $\lambda / 10$. En interférométrie hétérodyne, une résolution de $\lambda / 1000$ est annoncée et on parvient sans difficulté à $0,1 \mathrm{~nm}$. De même, en ellipsométrie et réflectométrie, une résolution "perpendiculaire " inférieure à une monocouche atomique est couramment signalée.

- Mais en microscopie la qualité de l'image dépend essentiellement de la résolution latérale, parallèle au plan de l'objet. Cette résolution a une limite théorique qui a été parfaitement définie à la fin du XIX ${ }^{\mathrm{e}}$ siècle par Abbe et Rayleigh [1]. Pour être vus comme deux points images résolus, deux points objets $A$ et $B$ doivent être séparés par une distance $\delta_{A B}$ telle que :

$$
\delta_{\mathrm{AB}} \geq \frac{1,22 \lambda}{\mathrm{n} \sin (\mathrm{u})}
$$

Dans cette formule, $\lambda$ est la longueur d'onde de la lumière utilisée, $n$ est l'indice du milieu dans lequel est "immergé " l'objet et u la demi-ouverture angulaire de l'objectif. La microscopie traditionnelle utilise de la lumière visible $(0,4 \mu \mathrm{m}<\lambda<0,8 \mu \mathrm{m})$. Aussi, même avec un objectif très ouvert $(\sin (u)=1)$ les images auront une résolution latérale théorique qui ne peut être inférieure à $0,25-0,4 \mu \mathrm{m}$. Dans la pratique, à cause des défauts résiduels des lentilles (aberrations géométriques et chromatiques), cette limite est habituellement aux environs d'un micromètre et exceptionnellement de $0,5 \mu \mathrm{m}$.

En utilisant des ondes électromagnétiques de plus courte longueur d'onde, ultraviolet ou même rayons $X$, la limite de résolution théorique pourrait être abaissée considérablement. Malheureusement pour ces longueurs d'onde la construction des optiques et la correction de leurs aberrations posent de très grandes difficultés et ces microscopes ont des résolutions latérales pratiques moins bonnes que celle du microscope optique.

Pour augmenter la résolution latérale, les microscopes électroniques remplacent la lumière par des électrons, mais leur principe reste le même. Ils utilisent des lentilles magnétiques pour focaliser les faisceaux d'électrons. La longueur d'onde associée aux électrons étant très courte (inférieure à $10^{-10} \mathrm{~m}$ ) on peut résoudre aujourd'hui des structures atomiques, malgré de difficiles problèmes d'aberrations nécessitant l'emploi de sources haute tension et des lentilles magnétiques complexes.

Le microscope électronique est aujourd'hui un outil de laboratoire et un instrument courant dans l'industrie. Cependant il nécessite une préparation des échantillons (coupe mince et métallisation) et une observation sous vide. Notons également que le faisceau d'électrons est très énergétique et peut endommager les échantillons, ce qui rend cette microscopie incompatible avec de nombreuses applications

L'optique nécessite moins de préparation d'échantillon, pas de métallisation et la lumière est moins endommageante que les électrons pour beaucoup d'échantillons. De plus l'optique peut apporter des méthodes d'analyse très puissantes comme la fluorescence, la polarisation et la spectroscopie.

Aussi, pour de nombreuses applications de recherches ou même industrielles il est très important de développer une méthode optique d'imagerie et d'analyse ayant une résolution latérale nettement en dessous du micron. La microscopie optique confocale [2] permet de gagner un facteur deux en résolution latérale et permet une véritable tomographie à trois dimensions des objets grâce à sa faible profondeur de champ. Mais il a fallu changer complètement le principe des microscopes pour améliorer sérieusement la résolution.

En microscopie, une révolution a eu lieu au début des années 1980. En inventant le microscope tunnel électronique [3] (Scanning Tunneling electronic Microscope: STM), Binning Rohrer et Gerber vont donner naissance à une nouvelle microscopie : la microscopie 
en champ proche ou à sonde locale. Cette découverte va valoir à leur auteurs le Prix Nobel en 1986 et elle va susciter la création de nombreux microscopes.

Ces microscopes sont fondés sur un principe complètement différent, ils n'utilisent pas de lentilles. Ils utilisent une sonde appropriée pour " éclairer " ou aller chercher l'information au voisinage immédiat de l'objet. En microscopie traditionnelle l'image est obtenue instantanément grâce à l'objectif, les microscopes à sonde locale sont des microscopes à balayage, l'image est construite point par point en déplaçant la sonde successivement sur tous les points de l'objet. Si la sonde est métallique et mesure un courant tunnel on obtient le STM. $\mathrm{Si}$ la sonde mesure une force on obtient un microscope à force atomique (Scanning Atomic Force Microscope : AFM [3]). Lorsque la sonde émet ou détecte de la lumière on obtient un microscope optique en champ proche dont le sigle générique est SNOM (Scanning Near-field Optical Microscope). Il existe bien d'autres microscopes à sonde locale mais leur développement est moins avancé que les trois précédents : microscope à capacité, microscope à sonde de Kelvin, microscope thermique

En fait, le principe de la microscopie optique à sonde locale avait été donné plus de 50 ans auparavant et en optique. Dans un article prophétique de E.H. Synge [4] qui suggère d'utiliser une nano-ouverture dans un écran métallique comme sonde. Cet article n'eut aucune suite et il faut attendre 1972 pour que les recherches redémarrent. Pour des raisons technologiques évidentes les premières expériences sont en onde radio [5] puis en infrarouge [6]. Il faudra attendre l'invention du STM pour que naissent les premières réalisations en optique. Le premier microscope en champ proche par transmission (Scanning Near field Optical Microscope" : SNOM) fut réalisé en 1983 au Laboratoire d'I.B.M. Zurich par D.W. Pohl, W. Denk., U. Dürig. [7], et par A. Lewis, M. Isaacson, A. Murray, A. Harootunian [8] à l'université Cornell aux Etats Unis.

\section{DIFFÉRENTES CONFIGURATIONS}

Depuis, de nombreux appareils ont été conçus, réalisés et ont obtenu des images. Dans ce paragraphe, tout en continuant cette rapide description historique, nous présenterons le principe des configurations les plus répandues et qui ont donné les meilleurs résultats tout en donnant des critères de classification.

D'abord, comme en microscopie classique il faut distinguer les microscopes travaillant en transmission ou par réflexion suivant la façon dont l'objet est éclairé.

Mais la spécificité des microscopes en champ proche est la façon dont la sonde est utilisée. Il y a trois modes de fonctionnement : le mode illumination, le mode collection et le mode perturbation.

Dans le mode illumination, la sonde est utilisée comme une nano-source illuminant l'objet en champ proche. Dans le mode collection, la sonde sert de collecteur de lumière pour capter le champ proche au voisinage de l'objet. Dans le mode perturbation la sonde ne sert ni à éclairer, ni à capter la lumière, mais sa présence au voisinage de l'objet modifie les conditions aux limites ce qui se répercute sur l'intensité d'un signal optique. Le principe des diverses configurations est représentée sur la figure 1

Les premières expériences étaient fondées sur le principe du microscope proposé par Synge, la sonde était une petite ouverture dans un écran métallique. En 1984, D.W. Pohl, W. Denk. et M. Lanz [9] ont proposé d'utiliser comme sonde une pointe : ils ont réalisé un microscope travaillant en transmission dans le mode illumination, la sonde est une nanoouverture à l'extrémité d'une fibre optique métallisée (fig. 1a). Une micropipette est utilisée dans le même but par l'équipe américaine [10]. Le premier microscope fonctionnant en transmission et en mode collection est réalisé en 1987 par Betzig et al. [11]. En 1989 un 


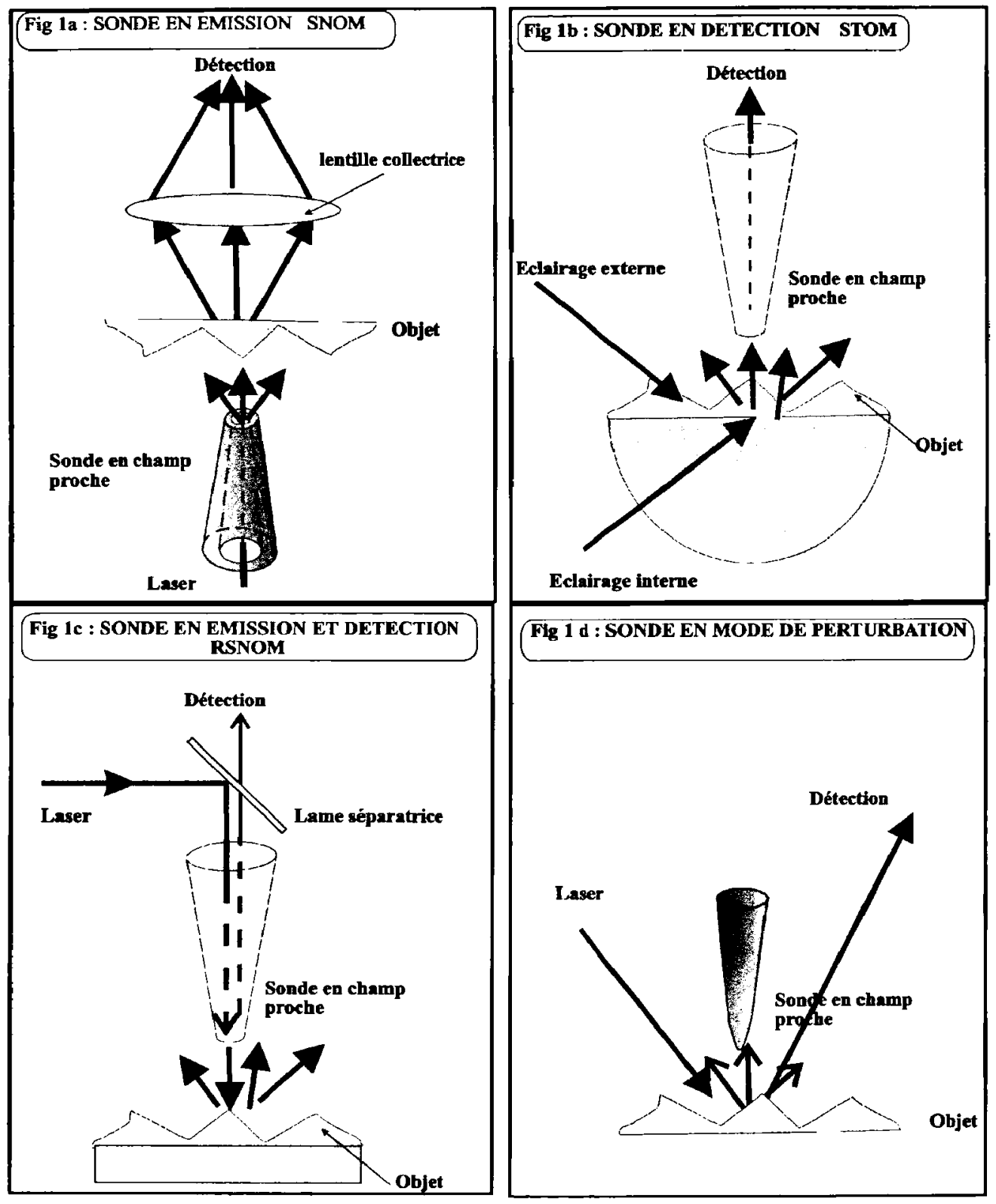

Figure 1 : Les principales configurations de microscopes optiques à sonde locale 
nouveau dispositif de microscopie optique en champ proche a été proposé par trois équipes Reddick et al.[12], Courjon et al. [13], De Fornel et al. [14]. Il s'agit d'un véritable Microscope Tunnel Optique appelé STOM (Scanning Tunneling Optical Microscope) ou PSTM (Photon Scanning Tunneling Microscope) par analogie avec le Microscope Tunnel Electronique. Ce microscope fonctionne en transmission mais l'objet est éclairé en réflexion totale interne ce qui produit sur la surface de l'objet une onde évanescente de Fresnel. Dans les premières applications de ce microscope tunnel optique, la sonde est l'extrémité d'une fibre optique " taillée " en pointe et utilisée telle quelle sans métallisation (fig. lb).

Les microscopes précédents travaillent en transmission, ce qui limite le champ de leurs applications. En 1989 M. Spajer et al. [15] ont développé un microscope optique en champ proche travaillant en réflexion où la pointe est utilisée à la fois en modes illumination et collection : la pointe est l'extrémité d'une fibre optique, elle sert, à l'aller, à éclairer l'objet en champ proche et, au retour, à collecter la lumière diffractée (fig. lc). Par la suite, nous désignerons ce microscope sous le sigle de RSNOM (Reflection SNOM).

Les microscopes SNOM et le STOM ont prouvé qu'ils pouvaient permettre d'obtenir des images avec une résolution latérale bien en dessous de celle donnée par le critère de Rayleigh [16-17]. La limite actuelle est difficile à préciser, elle dépend des objets et surtout du choix du critère de résolution, on peut estimer qu'elle est meilleure que $35 \mathrm{~nm}$ [17] et que les $15 \mathrm{~nm}$ ont été atteints [16].

Cette résolution n'est pas encore atteinte par le microscope fonctionnant en réflexion. En effet pour cette dernière technique la lumière transite deux fois par la pointe ce qui affaiblit considérablement le signal utile et diminue le rapport signal sur bruit limitant ainsi la résolution des images. Une résolution de $100 \mathrm{~nm}$ est signalée pour le RSNOM.

Très récemment on a assisté à un gain spectaculaire en résolution grâce au développement des microscopes fonctionnant en mode perturbatif. En 1992 Specht et al. [18] ont proposé un microscope en champ proche où une pointe métallique est utilisée pour perturber un plasmon de surface. Cette modification est observée en champ lointain par les modifications apportées sur l'intensité du faisceau réfléchi. L'équipe de A.C. Boccara [19] a développé un microscope en réflexion fonctionnant en perturbant un spot lumineux par une pointe et en captant la lumière diffractée par la pointe (fig. ld). Mais le principe de ce mode de fonctionnement pour la microscopie en champ proche se trouve déjà en 1990 dans un brevet Américain de Wickramasinghe et Williams [20]. Une pointe métallique très fine perturbe par son interaction le champ diffusé par l'objet. La détection en champ lointain de cette perturbation, par un dispositif interférométrique de grande sensibilité fournit un signal optique contenant de l'information sur la topographie ou les propriétés diélectriques locales de l'objet. Les premiers résultats datent de 1995 et les images obtenues montrent sans ambiguïté une résolution de $1 \mathrm{~nm}$ [21].

Il existe bien d'autres configurations que celles représentées sur la figure 1. Le SNOM peut également fonctionner en mode collection. Récemment D. Pohl a montré l'avantage d'utiliser une configuration tunnel optique où la sonde est en mode émission. Il s'agit d'un véritable STOM inversé (ISTOM) qui fonctionne en inversant le sens de propagation de la lumière dans une configuration STOM. On peut également utiliser un éclairage externe pour le microscope fonctionnant en réflexion (fig lc).

\section{PRINCIPE DE FONCTIONNEMENT}

Comme nous venons de le décrire, beaucoup de configurations ont été essayées en microscopie optique en champ proche. Mais elles utilisent toutes le même principe de fonctionnement : des ondes évanescentes sont transformées en ondes homogènes. 
Dans ce chapitre nous allons décrire les grandes lignes de la théorie. Faute de place nous utiliserons une théorie scalaire où les propriétés vectorielles et de polarisation de la lumière sont ignorées.

Le critère d'Abbe-Rayleigh est une limite théorique qui fut longtemps considérée comme infranchissable. De plus il est souvent démontré à l'aide des relations d'incertitude d'Heisenberg, ce qui renforce son caractère de limite inviolable. En fait, la microscopie optique en champ proche est en accord avec l'expression habituelle des relations d'incertitude et elle réussit à aller au delà de la limite de résolution des microscopes classiques en champ lointain en utilisant des ondes évanescentes.

\subsection{Onde évanescente}

La lumière est une onde électromagnétique vérifiant les équations de Maxwell. Pour une onde monochromatique, de longueur d'onde $\lambda=2 \pi \mathrm{c} / \omega$, se propageant dans le vide, on peut démontrer que ces équations impliquent que le champ électrique vérifie l'équation d'Helmholtz :

$$
\Delta E(x, y, z)+\frac{\omega^{2}}{c^{2}} E(x, y, z)=0
$$

Une onde plane est de la forme $E(x, y, z)=E(\vec{r})=E_{0} e^{i \vec{k} \cdot \vec{r}-i \omega t}(i=\sqrt{-1})$. Si cette onde est solution de l'équation d'Helmholtz, son vecteur d'onde vérifie l'équation de dispersion :

$$
\overrightarrow{\mathbf{k}}^{2}=\overrightarrow{\mathbf{k}} \cdot \overrightarrow{\mathbf{k}}=\mathrm{k}_{\mathrm{x}}^{2}+\mathbf{k}_{\mathrm{y}}^{2}+\mathbf{k}_{\mathrm{z}}^{2}=\frac{\omega^{2}}{\mathrm{c}^{2}}
$$

Une onde plane homogène a un vecteur d'onde réel, elle se propage sans atténuation dans la direction du vecteur d'onde. Pour une telle onde chaque composante a une longueur inférieure au module du vecteur. Par exemple $k_{x} \leq \frac{\omega}{c}=\frac{2 \pi}{\lambda}$.

Mais il existe aussi des ondes planes avec des vecteurs d'onde complexe. Dans ce cas on pose $\vec{k}=\vec{k}^{\prime}+i \vec{k}^{\prime \prime}$ où $\quad \vec{k}^{\prime}$ et $\vec{k}^{\prime \prime}$ sont deux vecteurs réels. Il s'agit d'une onde se propageant selon $\vec{k}^{\prime}$ sans atténuation mais elle est atténuée exponentiellement selon $\vec{k}^{\prime \prime}$. Il est d'ailleurs facile de montrer que les directions de propagation et d'atténuation sont orthogonales. En effet l'équation de dispersion implique $\vec{k}^{\prime} \cdot \overrightarrow{\mathbf{k}}^{\prime \prime}=0$. En prenant l'axe des $\times$ selon $\overrightarrow{\mathbf{k}}^{\prime}$ et l'axe des $\mathbf{z}$ selon $\overline{\mathrm{k}}^{\prime \prime}, \mathrm{l}$ 'équation de dispersion implique que la composante selon $\mathrm{x}$ du vecteur d'onde est plus grande que $2 \pi / \lambda: k_{x}=\sqrt{\left(\frac{2 \pi}{\lambda}\right)^{2}-k_{z}^{2}}=\sqrt{\left(\frac{2 \pi}{\lambda}\right)^{2}+\left(k^{\prime \prime}\right)^{2}} \geq \frac{2 \pi}{\lambda}$

La longueur caractéristique $L$, de l'atténuation en amplitude de l'onde, est définie par l'inverse de $k$ " : $L=1 / k$ ". Cette longueur d'atténuation est d'autant plus grande que $k$ " est petit, donc que $k$ ' est grand.

On nomme onde évanescente une telle onde. 


\subsection{Résolution d'un microscope optique}

On considère un objet créant une répartition de champ électrique avec des détails ayant une demi-largeur $\delta x$. dans l'espace direct. L'axe des $x$ est le plan parallèle à l'objet et l'axe des $z$ celui perpendiculaire. La lumière captée correspond à une distribution de vecteurs d'ondes de demi-largeur $\delta k_{\mathbf{x}}$. Les propriétés générales des transformées de Fourier font que ces deux largeurs vérifient la relation :

$$
\delta \mathbf{x} \delta \mathbf{k}_{\mathbf{x}} \geq 2 \pi
$$

La valeur précise du facteur numérique dans l'équation précédente dépend de la définition de ces largeurs (largeur à mi-hauteur, écart quadratique...).

Dans un microscope traditionnel, l'objectif est très loin de l'objet et seules les ondes homogènes peuvent être captées. En introduisant l'ouverture numérique du microscope, les ondes captées ont un vecteur d'onde dont la composante parallèle à l'axe des $\mathrm{x}$ est telle que $\mathrm{k}_{\mathrm{x}} \leq \frac{2 \pi}{\lambda} \sin (\mathrm{u})$. On choisit $\delta \mathrm{k}_{\mathrm{x}}=\frac{2 \pi}{\lambda} \sin (\mathrm{u})$. L'inégalité (4) conduit alors directement au critère de Rayleigh pour $\delta x$.

$$
\delta x \geq \frac{\lambda}{\sin (u)}
$$

La différence de valeur numérique entre les équations (1) et (5) provient simplement de la différence des définitions des largeurs et du fait que le critère de Rayleigh correspond à un éclairage incohérent, tandis que l'équation (5) est pour une lumière monochromatique donc cohérente, de plus, nous avons supposé dans ce paragraphe que nous travaillions dans le vide.

Pour augmenter la résolution d'un microscope, il faut diminuer $\delta x$ donc essayer d'augmenter $\delta \mathrm{k}_{\mathrm{x}}$. La microscopie optique en champ proche obtient ce résultat en utilisant des ondes évanescentes. En effet, nous avons vu qu'une onde évanescente, parce qu'elle décroît exponentiellement selon $z, a$ une composante selon $x$ du vecteur d'onde supérieure à $2 \pi / \lambda$ :

$$
\mathrm{k}_{\mathrm{x}} \geq \frac{2 \pi}{\lambda} \Rightarrow \delta \mathrm{k}_{\mathrm{x}} \geq \frac{2 \pi}{\lambda} \Rightarrow \delta \mathrm{x} \leq \lambda
$$

La démonstration précédente est due à J.M. Vigoureux [22], elle montre qu'en employant des ondes évanescentes on peut vérifier l'inégalité (4) tout en dépassant le critère de Rayleigh. La résolution latérale sera d'autant meilleure que l'on pourra obtenir des ondes évanescentes avec un $k_{\mathbf{x}}$ suffisamment grand.

\subsection{Efret tunnel optique}

La réflexion totale est le phénomène physique généralement associé à la création d'une onde évanescente. On considère la réflexion interne d'une onde plane homogène se propageant dans un milieu d'indice $n$ et subissant une réflexion sous l'angle $\theta$ sur un dioptre plan séparant le milieu $\mathrm{n}$ du vide (ou de l'air).

La traversée de l'interface plane ne change pas la valeur de la composante du vecteur d'onde parallèle au plan de l'interface. Le vecteur d'onde transmis a donc pour composante selon $\mathbf{x}$ : 


$$
\left(\mathrm{k}_{\mathrm{x}}\right)_{\text {incident }}=\left(\mathrm{k}_{\mathrm{x}}\right)_{\text {transmis }}=\mathrm{n}_{1} \frac{2 \pi}{\lambda} \sin (\theta)
$$

Si l'angle d'incidence est supérieur à l'angle limite , $\sin (\theta) \geq \sin \left(\theta_{1}\right)=\frac{1}{n_{1}}$, on obtient $\sin (\theta) \geq 1$ et on produit dans le vide une onde évanescente se propageant parallèlement au dioptre et atténuée perpendiculairement au dioptre. La partie complexe du vecteur d'onde transmis a pour valeur :

$$
k^{\prime \prime}=k_{z}=\frac{2 \pi}{\lambda} \sqrt{n_{1}^{2} \sin ^{2}(\theta)-1}
$$

Pour $\theta \geq \theta_{\mathbf{l}}$, malgré la création de l'onde évanescente, toute l'énergie incidente est réfléchie, c'est le phénomène de réflexion totale. Mais l'onde évanescente transmise existe bien. Elle peut exciter des molécules fluorescentes ou être captée par un autre dioptre ou une fibre optique, c'est l'effet tunnel optique (fig. 2). Pour une interface verre-vide $(n=1,5)$, la longueur caractéristique d'atténuation $L=1 / \mathrm{k}$ " est inférieure à la longueur d'onde mais demeure toujours supérieure à $\lambda / 10$. Pour capter l'onde évanescente il faut approcher l'atome, le dioptre ou l'extrémité de la fibre optique à une distance plus petite que $\mathrm{L}_{0}$, soit le dixième de micromètre pour de la lumière visible.

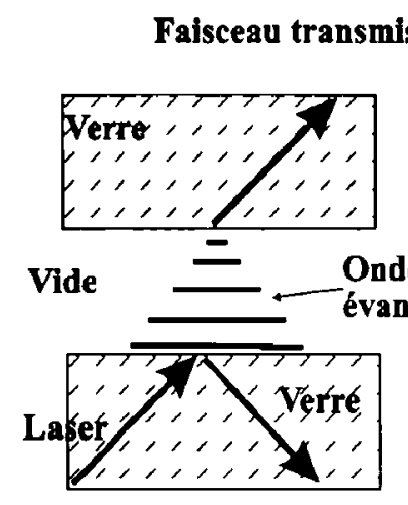

(a)

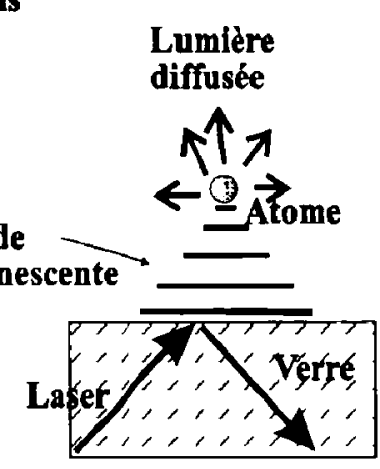

(b)

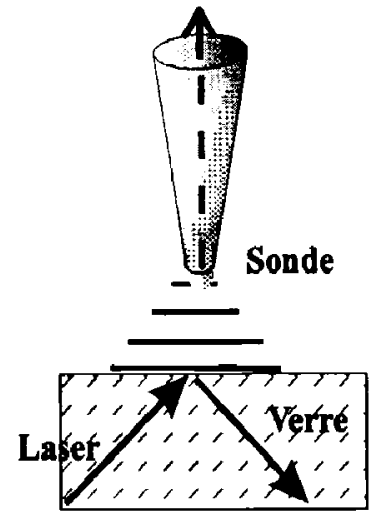

(c)

Figure 2 : Effet tunnel optique. Transformation d'une onde évanescente en onde homogènc par (a) un autre dioptre. (b) par diffusion sur un atome ou une molécule, (c) par diffraction sur une pointe.

Au delà de l'angle limite, la composante parallèle au plan de l'objet du vecteur d'onde transmis a bien une longueur supérieure à $2 \pi / \lambda$ et on peut ainsi espérer obtenir des images avec une résolution latérale au delà du critère de Rayleigh. Le microscope tunnel optique de Guerra [23] fonctionne sur ce principe. Il s'agit d'un microscope optique traditionnel à immersion mais l'échantillon est éclairé par des ondes évanescentes. De très belles images, très contrastées ont été ainsi produites. Leur résolution est meilleure que celle donnée par un éclairage traditionnel mais reste aux environs de $\lambda / 2$. En effet par ce procédé $k_{x}=n(2 \pi / \lambda) \sin (\theta)$ est bien plus grand 
que $2 \pi / \lambda$ mais le facteur multiplicatif est l'indice $n$ du verre. Par cette méthode on ne peut gagner qu'un facteur 2 au maximum.

Pour expliquer les grandes résolutions obtenues par les microscopes optiques en champ proche nous allons montrer que le phénomène de diffraction peut produire des ondes évanescentes avec des vecteurs d'ondes ayant une composante $k_{\mathrm{x}}$ très grande.

\subsection{Diffraction et onde évanescente. Spectre d'ondes planes.}

La théorie habituelle de la diffraction [1] utilise le principe de Huyghens Fresnel et exprime le champ diffracté en une somme d'ondes sphériques. La décomposition en spectre d'ondes planes [24] est plus intéressante car elle permet d'introduire des transformées de Fourier dans tous les problèmes de diffraction et pas seulement en diffraction de Fraunhofer. Elle est particulièrement adaptée à la discussion des problèmes de résolution spatiale car elle introduit le spectre spatial de l'objet.

Considérons dans le plan $z=0$ un objet de phase ou d'amplitude, transmettant ou réfléchissant une onde lumineuse monochromatique de fréquence circulaire $\omega=2 \pi / \lambda$. Cet objet diffracte l'onde incidente et l'on suppose connue l'amplitude $E(x, y, z=0) \exp (-i \omega t)$ du champ incident sur l'objet diffractant. Cette amplitude est une fonction de deux variables spatiales $\mathrm{x}$ et y et elle peut s'écrire sous la forme d'une transformée de Fourier à 2 dimensions :

$$
E(x, y, z=0)=\iint_{-\infty}^{+\infty} F\left(k_{x}, k_{y}\right) \exp \left[i\left(k_{x} x+k_{y} y\right)\right] d k_{x} d k_{y}
$$

Pour ne pas alourdir les futures discussions nous confondrons dans toute la suite le champ $E$ avec l'objet qui le crée. Ceci ne changera en rien les conclusions sur la résolution et le problème de la distance pointe-objet. Mais il est important de signaler que la détermination de E à partir de la connaissance de l'objet et du champ incident nécessite un gros travail théorique de calcul vectoriel de diffraction.

Le champ en $z=0$ possède un spectre de fréquences spatiales $F\left(k_{x}, k_{y}\right)$ et en général l'intégration dans l'équation précédente s'étend de $-\propto \dot{a}+\propto$ sur $\mathbf{k}_{\mathbf{x}}$ et $\mathrm{k}_{\mathrm{y}}$. Les propriétés des transformées de Fourier sont bien connues. Les grandes structures de l'objet vont contribuer à de basses fréquences spatiales et les détails fins à de hautes fréquences. Si l'objet a une largeur $\delta \mathrm{x}(\delta \mathrm{y})$, le spectre $\mathrm{F}\left(\mathrm{k}_{\mathrm{x}}, \mathrm{k}_{\mathrm{y}}\right) \mathrm{a}$ une largeur $\delta \mathrm{k}_{\mathrm{x}}\left(\delta \mathrm{k}_{\mathrm{y}}\right)$. Ces largeurs étant reliées par les relations : $\delta x \delta k_{x} \geq 2 \pi\left(\delta y \delta k_{y} \geq 2 \pi\right)$ exprimant le fait que plus l'objet est petit, plus son spectre $s$ 'étend dans les hautes fréquences

Grâce à cette introduction de la transformation de Fourier, il est facile d'obtenir l'expression du champ en tout point de l'espace $z>0$ :

$$
E(x, y, z)=\iint_{-\infty}^{+\infty} F\left(k_{x}, k_{y}\right) \exp \left[i\left(k_{x} x+k_{y} y\right)\right] \exp \left[i z \sqrt{\left(\frac{\omega}{c}\right)^{2}-k_{x}^{2}-k_{y}^{2}}\right] d k_{x} d k_{y}
$$

On vérifie facilement que l'équation précédente est bien solution de l'équation d'Helmholtz et qu'en $z=0$ elle redonne bien l'expression (8). Donc, si le champ diffracté est connu en $z=0$, l'équation (9) foumit une expression rigoureuse du champ diffracté en tout point de l'espace $(z>0)$. Pour interpréter l'équation (9), on introduit le vecteur 
$\vec{k}=\left(k_{x}, k_{y}, k_{z}\right)$, avec $k_{z}=\sqrt{\left(\frac{\omega}{c}\right)^{2}-k_{x}^{2}-k_{y}^{2}}$. L'intégrale (9) peut s'écrire sous la forme :

$$
E(x, y, z)=\iint_{-\infty}^{+\infty} F\left(k_{x}, k_{y}\right) \exp [i \vec{k} \cdot \vec{r}] d k_{x} d k_{y}
$$

le vecteur $\overrightarrow{\mathrm{k}}$ vérifie bien l'équation de dispersion (3). Le champ diffracté peut donc s'exprimer comme une somme d'ondes planes, chaque onde plane ayant une amplitude $F\left(k_{x}, k_{y}\right)$ et un vecteur d'onde $\overrightarrow{\mathbf{k}}$. Mais comme dans le champ en $\mathbf{z}=0$, les intégrations portent sur toutes les fréquences spatiales, les intégrales (9) et (10) comportent aussi bien des ondes homogènes $\left(k_{z}\right.$ réel) que des ondes évanescentes avec $\mathbf{k}_{\mathbf{z}}$ imaginaire pur.

Les basses fréquences spatiales $\left(k_{x}^{2}+k_{y}^{2} \leq \frac{\omega^{2}}{c^{2}}\right)$ donnent des ondes homogènes se propageant sans atténuation, les hautes fréquences spatiales $\left(k_{x}^{2}+k_{y}^{2}>\frac{\omega^{2}}{c^{2}}\right)$ donnent des ondes évanescentes s'attenuant exponentiellement. La diffraction permet de créer des ondes évanescentes ayant des vecteurs d'onde $k_{x}$ ou $k_{y}$ très grands.

Le champ en $z>0$ s'écrit sous la forme d'une transformée de Fourier à 2 dimensions dont le spectre est $F\left(k_{x}, k_{y}\right) \exp \left[i z \sqrt{\left(\frac{\omega}{c}\right)^{2}-k_{x}^{2}-k_{y}^{2}}\right]$.

Le spectre du champ est donc modifié, filtré, par la propagation du champ selon z. Pour les basses fréquences spatiales ce filtrage est un simple déphasage. Il n'y a pas dans ce cas perte d'information mais ces déphasages donnent des modifications des champs diffractés caractéristiques de la diffraction de Fresnel ou de la diffraction de Fraunhofer. Par contre, pour les hautes fréquences spatiales, l'exponentielle est de module inférieur à 1 et il y a atténuation des hautes fréquences spatiales. Cette atténuation est d'autant plus élevée que la fréquence spatiale est élevée. La propagation selon $z$ du champ diffracté est donc un filtre passe-bas.

\subsection{Principe de fonctionnement des microscopes optiques en champ proche}

Les explications des chapitres précédents permettent de comprendre le principe de fonctionnement des microscopes optiques en champ proche

Il est plus facile de commencer par le microscope où la sonde est utilisée en mode de détection. Le champ diffracté par un objet beaucoup plus grand que la longueur d'onde ne contient essentiellement que des basses fréquences spatiales, qui vont donner des ondes homogènes se propageant sans atténuation. Pour de tels objets, une lentille en champ lointain peut capter toutes ces ondes et on peut reconstituer une image correcte de l'objet. Quand l'objet diminue, son spectre spatial comprend des parties significatives en hautes fréquences qui ne pourront être captées en champ lointain. L'image de cet objet va être dégradée. Quand l'objet est plus petit que la longueur d'onde tout son spectre est en hautes fréquences et on ne peut résoudre cet objet à l'aide d'un objectif de microscope. 
Par contre en faisant travailler une sonde en champ proche, immergée dans les ondes évanescentes correspondant aux hautes fréquences de l'objet, on peut capter ces ondes et recueillir l'information qu'elles transportent sur les détails fins de l'objet. Dans ce type de microscope, la sonde sert à convertir par diffraction ces ondes évanescentes en ondes propagatives guidées ensuite vers le détecteur.

Pour comprendre le principe de fonctionnement d'un microscope où la sonde fonctionne en mode émissif, il suffit d'appliquer le principe de réciprocité (principe du retour inverse de la lumière) au raisonnement précédent. La lumière incidente est guidée de la source à la pointe. Cette dernière est un objet très petit qui diffracte le mode incident propagatif en un champ diffracté comportant beaucoup d'ondes évanescentes. En éclairant l'objet, en champ proche, par un tel champ, riche en hautes fréquences spatiales on obtient par diffraction des modes propagatifs pouvant être captés en champ lointain.

Les microscopes en mode émission ou détection fonctionnent sur le même principe, il suffit d'inverser les rôles de l'objet et de la sonde pour passer de l'un à l'autre. Il est alors facile de comprendre que les microscopes fonctionnant en mode de perturbation reposent sur le même principe.

\subsection{Distance sonde-objet et résolution}

En microscopie classique, la résolution dépend de l'ouverture numérique de l'objectif et de la longueur d'onde utilisée. En microscopie optique en champ proche, un paramètre fondamental influant sur la résolution est la distance sonde objet.

Pour les microscopes où la sonde fonctionne en mode de détection, le champ proche émis par l'objet peut s'exprimer sous la forme d'un spectre d'ondes planes (équations 9-10). Pour résoudre un détail de largeur $\delta$, il faut capter au moins la fréquence spatiale de vecteur d'onde transversal ayant pour longueur $k_{t}=\sqrt{k_{x}^{2}+k_{y}^{2}} \approx \frac{2 \pi}{\delta}$. Si $\delta$ est plus petit que $\lambda$, cette fréquence spatiale va donner une onde évanescente. La longueur de décroissance de cette onde est donnée par le module de $k_{z}$ :

$$
L=\frac{1}{\left|k_{z}\right|}=\left(k_{x}^{2}+k_{y}^{2}-\left(\frac{2 \pi}{\lambda}\right)^{2}\right)^{-1 / 2} \approx\left(\frac{4 \pi^{2}}{\delta^{2}}-\frac{4 \pi^{2}}{\lambda^{2}}\right)^{-1 / 2} \approx \frac{\delta}{2 \pi}
$$

Ainsi, pour $\delta<\lambda$ (pratiquement $\delta<\lambda / 3$ ) cette longueur de décroissance devient à peu près indépendante de la longueur d'onde et ne dépend que de la taille du détail à résoudre. On peut faire une semblable discussion pour le microscope en mode émissif ou en mode perturbatif.

En conclusion, pour des objets plus petits que la longueur d'onde, la résolution des microscopes optiques en champ proche est pratiquement indépendante de la longueur d'onde utilisée et elle dépend de la distance sonde-objet. Plus on veut résoudre un détail petit, plus il faut approcher l'objet à une petite distance. Pour résoudre un détail de $100 \mathrm{~nm}$, il faut que la sonde soit à une distance de $20 \mathrm{~nm}$. Ce qui va impliquer pour les microscopes optiques en champ proche l'utilisation de céramiques piézo-électriques pour contrôler la position des sondes avec cette précision. 


\section{PROBLÈMES TECHNIQUES ET LEURS SOLUTIONS}

Si le principe des microscopes optiques en champ proche est simple, de nombreux problèmes techniques sont à résoudre pour passer du principe à la réalisation. Nous allons rapidement les passer en revue et donner les solutions qui ont été proposées pour les résoudre. Nous commencerons par les problèmes techniques communs à toutes les microscopies en champ proche. Dans toute la suite le plan moyen de l'objet, plan transversal, sera le plan (x-y). L'axe $z$ est donc la direction perpendiculaire au plan de l'objet, c'est la direction d'approche de la sonde.

\subsection{Dispositif de balayage}

Comme tous les microscopes à sonde locale, les microscopes optiques en champ proche utilisent des céramiques piézo-électriques (PZT: Piezo-electric Transducer) pour placer et déplacer la pointe dans les trois dimensions de l'espace. Ces dispositifs sont commercialisés avec leur électronique de commande. Vu les résolutions obtenues actuellement en optique, une résolution du $\mathrm{nm}$ en $(x, y)$ semble suffisante. Une résolution de $0,1 \mathrm{~nm}$ est nécessaire en $\mathrm{z}$. La qualité du dispositif de balayage dépend de celle des céramiques (linéarité et faible hystérésis), mais l'électronique joue un rôle fondamental sur les résultats obtenus; elle permet une compensation de l' hystérésis et des non-linéarités et doit avoir un excellent rapport signal/bruit ( $1 \mathrm{mV}$ sur $100 \mathrm{~V}$ de tension). Le dispositif avec 3 céramiques en trièdre orthonormé est peu utilisé en optique. Des configurations en tube ou en cylindre sont plus utilisées. La surface cylindrique est métallisée par quadrant permettant une translation commandable en $x, y$ et $z$ avec un seul cylindre.

Pour l'approche grossière de la pointe vers l'échantillon, des dispositifs annexes sont nécessaires (vis micrométriques, moteur pas à pas ou PZT de grandes amplitudes). Cette approche doit être contrôlée optiquement par une lunette ou un microscope de visée.

Les propriétés du PZT dépendent de la température; un bon contrôle de la température des expériences peut s'avérer nécessaire.

\subsection{Filtrages des vibrations parasites}

C'est un problème technique commun à toutes les microscopies mais plus particulièrement aux microscopies à sonde locale.

Le microscope proprement dit doit être très compact de façon à ce que les fréquences de résonance soient hautes et éloignées des fréquences de vibrations parasites.

Le microscope doit être découplé le plus possible des sources de vibrations extérieures. Les vibrations mécaniques, généralement de basses fréquences, peuvent être éliminées par une table antivibration, en suspendant le microscope, en posant le dispositif sur un empilement de plaques métalliques séparées par des joints élastiques. Les vibrations acoustiques sont également gênantes. Une simple hotte en verre ou en plastique permet d'en éliminer une grande partie.

La qualité des images et leur résolution dépend de la qualité des solutions apportées aux problèmes précédents. Mais les points qui vont suivre sont spécifiques des microscopes optiques et demandent à être traités avec encore plus de précautions. 


\subsection{Sources de lumière}

La source de lumière peut être quelconque (source conventionnelle, photodiode, diode laser, laser). On utilise généralement un laser à quelques $\mathrm{mW}$ de puissance. Des flux supérieurs pourraient être nécessaires pour les applications spectroscopiques, mais il faut faire attention aux problèmes d'échauffement destructif de l'échantillon pour le mode en collection ou de la pointe pour le mode en illumination.

Le choix de la longueur d'onde n'est pas fondamental, contrairement à la microscopie traditionnelle la longueur d'onde employée a peu d'influence directe sur la résolution des images.

Par contre la stabilité en intensité des sources employées est fondamentale pour la qualité et la résolution des images.

\subsection{Détection}

Elle ne pose aucun problème insurmontable. Les expériences de microscope en champ proche sont suffisamment lumineuses pour permettre l'utilisation de cellules photoélectriques. Un photomultiplicateur, surtout s'il est refroidi, améliore le rapport signal sur bruit. Les cellules CCD à très faible bruit ont été employées dans des expériences de spectroscopie où le signal détecté est très faible.

Les expériences peuvent être réalisées avec des sources lumineuses continues. Mais il peut être utile, pour améliorer le rapport signal sur bruit, de moduler le faisceau lumineux et d'utiliser une détection synchrone.

\subsection{Asservissement de la position de la pointe}

Les céramiques piézo-électriques assurent la mise en place de la pointe, mais il est nécessaire de stabiliser cette position et de la contrôler par une boucle d'asservissement.

Ces remarques sont générales pour toutes les microscopies à sonde locale, mais des problèmes sont spécifiques à l'optique.

En effet, en STM, le courant tunnel qui décroît exponentiellement lorsque l'on éloigne la pointe peut servir de signal de contrôle de l'asservissement. L'équivalent optique du STM est le microscope STOM, le signal lumineux capté par le film décroît également exponentiellement et peut servir à asservir la fosition de la pointe.

En SNOM ou pour le RSNOM, le signal optique ne varie pas de façon monotone, il est impossible d'utiliser le signal lumineux pour contrôler la position de la pointe et un autre signal d'une autre nature physique doit être employé.

Pour les microscopes utilisant une pointe métallique et travaillant sur des objets métalliques on peut réaliser en même temps que l'expérience optique une expérience d'effet tunnel électronique, le courant tunnel sert pour l'asservissement. Bien évidemment ce type d'asservissement est utilisé pour les microscopes en mode de perturbation utilisant une pointe STM.

On a aussi proposé de coupler un microscope optique en champ proche avec un AFM [25, 26]. La pointe servant de sonde de force et de pointe optique, la mesure sert alors à asservir la position de la pointe. Ce type de microscope est intéressant, car il fournit simultanément une image en optique de l'objet et une image de force. De plus il permet d'utiliser des pointes AFM commercialisées donc de nature et de forme bien connues. 
Un autre dispositif d'asservissement, qui est aujourd'hui très utilisé, est le " Shear-force " (force de cisaillement) [27]. Pour un microscope travaillant à l'air, une couche liquide, d'eau plus ou moins polluée par de l'adsorption, existe toujours entre la pointe et l'objet. La force de liaison provoquée par ce film liquide dépend fortement de la distance pointe-surface. Pratiquement, on fait vibrer la pointe parallèlement à la surface de l'objet grâce à une céramique piézo-électrique supplémentaire. La fréquence de vibration se situe entre 1 et 10 $\mathrm{kHz}$. L'amplitude de la vibration dépend de l'amortissement, donc de la force de cisaillement entre la pointe et le film liquide et ceci de façon monotone : l'amortissement augmenterait fortement quand la pointe est très près de la surface. Un dispositif optique annexe, éclairant les pointes transversalement, mesure cette amplitude de vibration. On peut ainsi asservir la position " en $\mathrm{z}$ " de la pointe grâce à ce signal " shear-force ".

Ce type d'asservissement semble être facile à mettre en oeuvre, fiable et robuste.

Ces asservissements par mesure de force (AFM ou Shear-force) intéressent beaucoup les expérimentateurs car l'image de force obtenue simultanément avec l'image optique devrait refléter étroitement la topographie de l'objet. Ce résultat est faux en toute rigueur et devrait être utilisé avec précautions : la force et ses variations dépendent a priori de la nature de la pointe et des modifications locales de l'objet. Des images avec des inversions de contraste (creux à la place de bosses) en image AFM ou Shear-force ont été produites expérimentalement.

Suivant la façon dont est réglé l'asservissement, trois types d'images peuvent être obtenues. Des images à " hauteur constante ", si l'asservissement maintient la pointe à distance constante du plan moyen de l'échantillon. Ces images sont des cartes de l'intensité détectée à une certaine hauteur au dessus de l'objet. Des images à " intensité constante " sont obtenues en maintenant à une valeur de consigne le signal optique détecté. Lorsque la pointe se déplace on maintient ce signal constant en faisant varier le réglage en z des PZT. Ces images à " intensité constante " sont des cartes de rugosités apparentes qu'il est important d'essayer de relier à la topographie de l'objet. En asservissant la position de la pointe à l'aide d'une mesure de force (A.F.M. ou Shear-force) on peut espérer maintenir la pointe à une distance constante de la surface de l'objet. Ce troisième mode de fonctionnement est utilisé car il permettrait de séparer le vrai signal optique, d'un signal optique créé par les variations de hauteur de l'échantillon. La remarque du paragraphe précédent montre qu'un travail d'interprétation des images est également nécessaire pour ce mode d'asservissement.

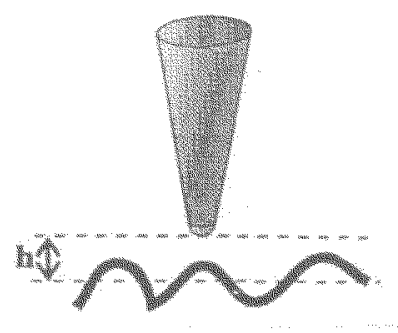

(a)

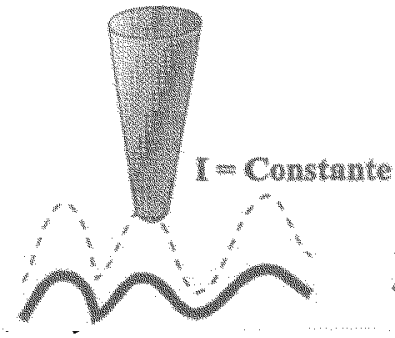

(b)

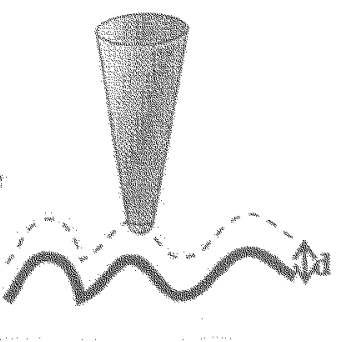

(c)

Figure 3 : différents modes d'asservissement de la position de la sonde. Selon le réglage de l'asservissement des céramiques piézo-électriques la sonde peut balayer la surface de l'objet selon 3 modes.

a) à distance constante du plan moyen de l'objet.

b) à signal détecté constant.

Comme le signal optique ne suit pas le profil de l'objet il peut être avantageux d'asservir à distance constante de la surface de l'objet (c). Cette distance est contrôlée par un système annexe (mesure de force). 


\subsection{Réalisation des sondes optiques}

Le problème technique le plus important des microscopes optiques en champ proche est la réalisation de la sonde optique.

\subsubsection{Fibre optique taillee en pointe}

Actuellement la quasi-totalité de ces microscopes utilisent une fibre optique taillée en pointe. La pointe sert de sonde locale et la fibre optique sert à guider la lumière de la source à la pointe en mode illumination ou de la pointe au détecteur en mode collection. On utilise généralement des fibres monomodes, de diamètre de cour d'environ $1 \mu \mathrm{m}$. La pointe peut être obtenue par deux procédés différents.

Le premier procédé consiste en une attaque chimique de l'extrémité de la fibre dans une solution à base d'acide fluorhydrique. La composition, la concentration de la solution ainsi que la durée de l'attaque détermine la forme de la pointe : son rayon de courbure à l'extrémité et l'angle du cône. La référence [28] détaille ce procédé de fabrication et présente quelques images de pointes.

Le second procédé est plus physique, il combine le chauffage (par un laser à $\mathrm{CO}_{2}$ ou un arc électrique) et l'étirement mécanique de la fibre. La forme exacte de la pointe est là aussi fortement liée aux détails du processus de fabrication. La référence [29] présente une étude assez systématique de cette méthode et compare les différentes pointes obtenues.

Les deux procédés ou leur combinaison parviennent à des résultats comparables. On peut fabriquer de façon contrôlée et reproductible des pointes effilées de forme conique et dont le rayon de courbure de l'extrémité ne semble pas pouvoir être plus petit que $20-30 \mathrm{~nm}$.

Des images à haute résolution ont été obtenues en utilisant des pointes sans autre traitement: pointe diélectrique nue ("uncoated tip"). Par contre d'autres laboratoires ont préféré métalliser l'extrémité de la pointe. De telles pointes sont obtenues par étirement à chaud puis métallisées sous vide par de l'aluminium. La métallisation a lieu par évaporation sous vide. L'évaporation du métal, généralement de l'aluminium, a lieu de biais sur une pointe tournant lentement. De cette façon l'ultime extrémité de la pointe reste plane et non métallisée : réalisant ainsi l'idée de Synghe [4], une petite ouverture dans un écran métallique. Ce type de pointe est commercialisée, le diamètre de l'ouverture semble être compris entre 20 et $200 \mathrm{~nm}$.

\subsubsection{Pointe A.F.M.}

Certains types de microscopes à force atomique (AFM) utilisent des pointes diélectriques qui sont directement fixées sur le levier servant à mesurer la force. Les pointes sont généralement en nitrure de silicium qui est un matériau transparent, elles peuvent donc être également utilisées comme sondes optiques. Ces ensembles pointe-levier sont commercialisés, ce qui a l'avantage de fournir des pointes suffisamment fines mais surtout de structure et de forme connues et reproductibles. Quelques équipes [25-26] ont donc proposé de réaliser des microscopes mixtes AFM-SNOM. La partie AFM sert à contrôler la distance pointe-surface et à former une image topographique de l'objet. La pointe sert aussi de sonde optique, elle est généralement utilisée en mode détection. La figure (4) prësente le schéma de principe d'un microscope de ce type. Le microlevier porte une pointe à son extrémité. La fibre optique clivée est juste au dessus du levier. Elle capte le champ diffracté par la pointe. Elle sert aussi à véhiculer à l'aller et au retour le faisceau servant à déterminer la position de la pointe. 


\subsubsection{Pointes STM}

Le microscope en mode perturbatif n'a pas besoin d'une pointe transparente car la sonde optique ne sert pas à éclairer ni à détecter la lumière.

On peut donc utiliser une pointe STM métallique. Ces pointes, généralement en tungstène sont extrêmement fines (fine à l'échelle atomique) ce qui est certainement une des raisons de la très grande résolution obtenue par ces microscopes

\section{Fibre Optlque}

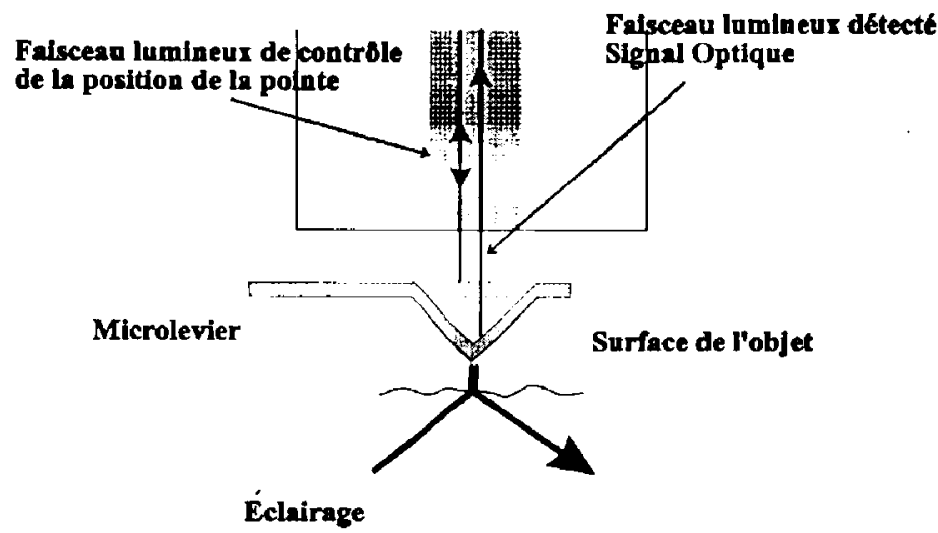

Figure 4 : Principe d'un microscope mixte AFM-STOM

\subsection{Autres pointes}

Afin d'améliorer l'efficacité lumineuse des sondes optiques utilisées en microscopie en champ proche d'autres types de sondes ont été proposées. La structure coaxiale avec une âme métallique au milieu du guide d'onde a été proposée par plusieurs groupes [30-31]. Elle se heurte à de difficiles problèmes de réalisation. Ces problèmes sont plus facilement résolus en infrarouge où ce type de sonde pourrait être développé.

Dans le même but $U$. Fischer [32] a proposé d'utiliser une pointe tétraédrique. Elle est construite en utilisant un coin d'une lame de microtome et en métallisant deux de ces faces. Une résolution de $1 \mathrm{~nm}$ aurait été atteinte récemment à l'aide de ce type de pointe.

Danzebrink et al. [33] ont obtenu une pointe en forme de coin en clivant un monocristal d'AsGa. L'utilisation de matériaux semi-conducteurs ouvre la voie à de nouvelles possibilités très intéressantes [34] : faire des pointes détectrices où la conversion lumière-électricité est faite au niveau de la pointe dans le champ proche ; ou inversement réaliser l'émission au niveau de la sonde optique. Des expériences très prometteuses ont égajement été réalisées avec un laser fibré [35] dont l'extrémité taillée en pointe selon les techniques usuelles servait de sonde SNOM. Le couplage avec l'objet modifie les conditions d'accord du laser. Ce dispositif semble être d'une grande sensibilité et pourrait avoir de futures applications pour le stockage de masse des données informatiques 
Il est également possible de faire croître, par ingénierie chimique, des molécules photosensibles à l'extrémité de la sonde optique. Ces molécules peuvent jouer un rôle d'amplification, de guidage du champ proche. Les réactions photochimiques étant dépendantes du $\mathrm{pH}$ ou du potentiel d'oxydoréduction, on disposerait ainsi par la voie de la microscopie optique à sonde locale, de sondes chimiques très utiles pour les applications biologiques [36].

\section{RÉSULTATS ET APPLICATIONS}

\subsection{Résultats en résolution}

De nombreuses équipes de par le monde maîtrisent parfaitement la technique des microscopes optiques en champ proche où la sonde est une fibre optique taillée en pointe et qui fonctionne en mode de détection ou en mode d'émission. La résolution ultime atteinte en 1996 semble être voisine de $15-20 \mathrm{~nm}$, que la pointe soit utilisée telle quelle ou métallisée.

La figure 5 présente des images d'un réseau bidimensionnel de plots métalliques de $40 \mathrm{~nm}$ de hauteur. La période est d'environ $300 \mathrm{~nm}$. La taille d'une image est de $2,25 \mu \mathrm{m}$ sur $2,25 \mu \mathrm{m}$. La lumière utilisée a pour longueur d'onde $\lambda=638 \mathrm{~nm}$, un tel réseau est invisible avec le meilleur microscope classique à cause de sa faible hauteur et de sa période bien en dessous de la longueur d'onde. Le microscope utilisé pour imager ce réseau est un microscope mixte AFM-STOM dont le principe est représenté sur la figure 4 mais qui a été rendu plus compact [37].

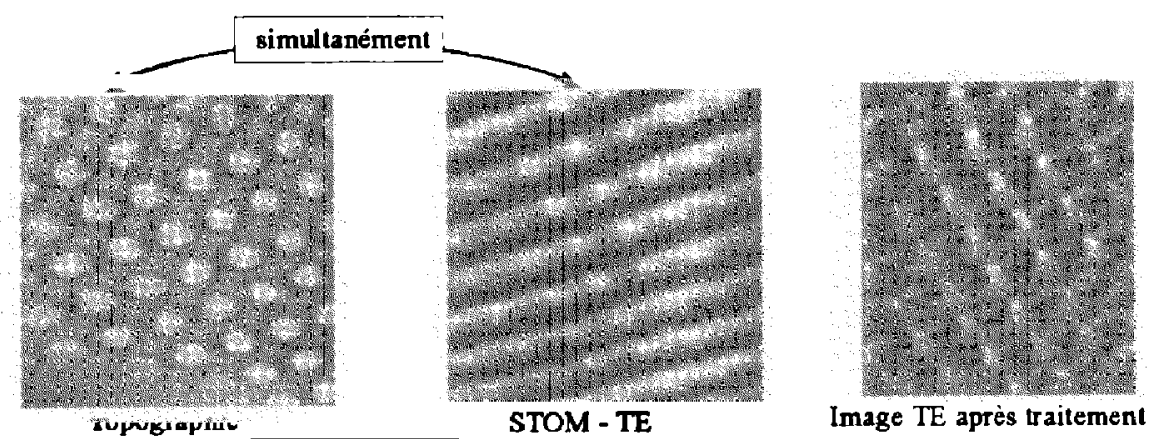

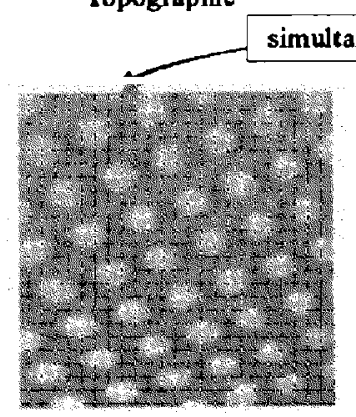

Topographie
STOM - TE

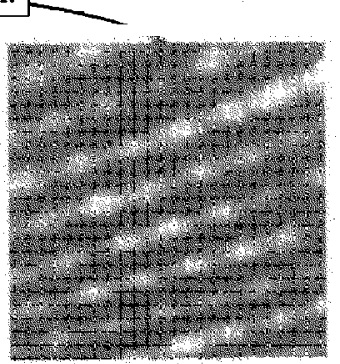

STOM - TM

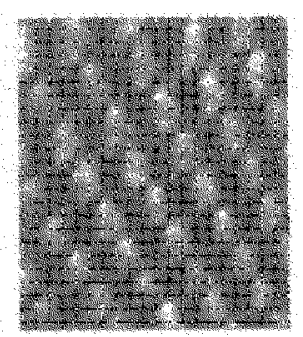

Image TM après traitement

Figure 5 : Comparaison des images AFM et STOM d'un réseau de plots métalliques [37]. 
La figure 5 compare une image topographique de l'objet obtenue avec l'AFM et une image optique obtenue simultanément au cours du balayage. En optique, deux images sont prises avec des polarisations différentes: TE (images supérieures) et TM (images inférieures). La polarisation TE est perpendiculaire au plan d'incidence et la polarisation TM est dans le plan d'incidence. La partie droite de la figure 5 présente les images optiques après traitement informatique. Par transformation de Fourier on peut estimer la résolution des images optiques meilleure que $50 \mathrm{~nm}$. $15-20 \mathrm{~nm}$.

La référence [16] contient des images obtenues en SNOM et démontrant une résolution de

Cette limite en résolution est certainement due à une contrainte technologique, le matériau de constitution des pointes (verre ou silice) ainsi que les procédés de fabrication semblent imposer un rayon de courbure de l'extrémité des sondes supérieur à 10-20 nm.

Comme nous l'avons signalé des images optiques avec une résolution de $1 \mathrm{~nm}$ ont été obtenues par un microscope fonctionnant en mode de perturbation. Cette haute résolution est certainement due à l'utilisation de pointes STM, fines à l'échelle atomique et à l'utilisation d'un dispositif interférométrique permettant une amélioration sensible du rapport signal sur bruit. Ces nouveaux résultats demandent à être confirmés mais ils sont très prometteurs et ils vont certainement relancer le développement de nouveaux appareils

A ce jour les microscopes optiques en champ proche ont prouvé que l'on pouvait en optique obtenir des images avec une résolution latérale bien en deçà du critère de Rayleigh. Mais la résolution est encore (pour combien de temps ?) moins bonne que celle du STM et de l'AFM. Cependant même avec une résolution limitée à $20 \mathrm{~nm}$ la microscopie optique en champ proche est promise à un grand développement car elle permet d'étendre dans le domaine nanométrique toutes les méthodes optiques d'analyse, de mesure et de fabrication et depuis deux ans le nombre de publications est en forte augmentation illustrant les diverses possibilités de cette nouvelle microscopie optique.

\subsection{Changement de longueur d'onde et spectroscopies}

Nous avons déjà signalé qu'en microscopie optique traditionnelle il est très difficile de changer de longueur d'onde. Les aberrations des lentilles sont corrigées facilement en lumière visible car on dispose de nombreux matériaux d'indices variés. Il n'en est pas de même en ultraviolet ou en infrarouge où n'existe souvent qu'un seul type de matériau transparent.

Ce problème est beaucoup moins grave en microscopie optique en champ proche. En effet, il suffit de trouver une sonde transparente pour la longueur d'onde utilisée. Ceci est beaucoup moins difficile que la détermination de combinaison optique corrigeant les aberrations. Des fibres optiques existent pour l'infrarouge et l'ultraviolet dans certaines gammes de longueurs d'onde. Comme les longueurs de fibre sont courtes, la fibre peut être partiellement absorbante.

Les microscopes fonctionnant en mode de perturbation peuvent facilement être utilisés en réflexion et ils n'exigent pas nécessairement de sonde transparente. Ce type de microscope doit permettre de changer de longueur d'onde sans aucune difficulté technique, autre que le choix de la source et du détecteur. Ainsi récemment $C$. Bocarra [38] a pu observer des détails de $0,015 \mu \mathrm{m}$ sur un film d'or éclairé par un laser à $\mathrm{CO}_{2}$ de longueur d'onde $10 \mu \mathrm{m}$.

Pour les applications en imagerie il peut être très utile d'observer un même objet en changeant la longueur d'onde afin de mettre en évidence des détails plus diffusants ou plus absorbants. La fluorescence spécifique de certaines parties de l'objet peut être aussi utilisée pour augmenter le contraste des images [39]. Les marqueurs fluorescents de biologie [39] 
peuvent être utilisés en champ proche et, dans ce cas, un simple filtre permet de séparer les parties marquées du reste de l'objet.

Mais en adjoignant un spectroscope à un microscope optique en champ proche on peut aller bien au delà de la simple imagerie : augmenter la résolution spatiale de toutes les méthodes de spectroscopie optique, et étendre ainsi dans le domaine nanométrique les possibilités d'analyse chimique.

L'obtention d'un spectre à partir d'un microscope optique en champ proche suppose que tout le dispositif fournisse assez de signal pour être analysé, ce qui suppose une optimisation de nombreux paramètres : une grande efficacité de la fibre optique, un bon couplage de la lumière captée vers le spectroscope, une très bonne luminosité du spectroscope et un détecteur très sensible. Ces problèmes ont été résolus par plusieurs équipes et des applications spectroscopiques sont publiées. Un SNOM spectroscopique [40] pouvant fonctionner sous vide et monté dans un cryostat fonctionnant de la température ambiante à celle de l'hélium liquide, a permis l'étude par spectroscopie, de structures à puits quantiques en physique du solide [41]. A ce jour la sensibilité des appareils est suffisante pour l'observation de molécules uniques [42], la spectroscopie de molécule unique [42] et la mise en évidence d'effets de sites par comparaison des spectres de molécules identiques mais situées à des endroits différents [43-44]. Des expériences de spectroscopie résolues dans le temps par mesure du temps de déclin de fluorescence ont également été effectuées en champ proche [45] et même sur des molécules uniques [46-47].

Piednoir et al. [48] ont utilisé un STOM pour obtenir les premiers spectres en champ proche dans l'infrarouge. Ils utilisaient une fibre en verre fluoré (coupure à $8 \mu \mathrm{m}$ ) et un détecteur $\mathrm{HgCdTe}$. La source fut d'abord une lampe Globar et finalement le rayonnement synchrotron de CLIO. Ils ont obtenu le spectre d'une photorésine et ont pu montrer les différences avec un spectre en champ lointain. La résolution spectrale était de $7 \mathrm{~cm}^{-1}$.

Dans un proche avenir on devrait assister également à la naissance de la spectroscopie Raman en champ proche. Les travaux préliminaires ont lieu dans plusieurs laboratoires.

\section{3 Étude locale de dispositifs optiques}

De nombreux dispositifs opto-électroniques ont maintenant des dimensions de l'ordre du micromètre et la microscopie optique à sonde locale (en mode détection) est un outil idéal pour aller sonder "in-situ" la structure des champs et vérifier le bon fonctionnement de ces dispositifs. Ce genre d'étude est souvent impossible en champ lointain ou foumit peu de renseignements

Les guides d'ondes optiques ont au dessus de leur structure des ondes évanescentes reflétant la structure des modes qui s'y propagent. Le SNOM en mode de détection permet de visualiser la structure des modes [49], de vérifier quels sont les modes vraiment injectés, de montrer des interférences inter-modales et le comportement du champ à une jonction

On a aussi proposé d'utiliser la sonde pour tracer la carte des champs émis par l'extrémité d'une fibre, d'un guide clivé, d'un laser à puits quantiques [48-49]. Récemment, $O$. Marti [51] a montré une nouvelle application au contrôle en fonctionnement d'une diode laser. La sonde optique est immergée dans le champ émis par la diode. Un spectroscope analyse la lumière émise ce qui permet de séparer les différents modes. La microscopie optique en champ proche permet ainsi d'obtenir une carte de la structure en mode du champ émis par la diode et d'étudier la répartition de ces modes en fonction du courant d'alimentation. 


\subsection{Photolithographie}

La photolithographie dans des résines photosensibles était une méthode utilisée dans les laboratoires de microélectronique pour graver des masques. La largeur des traits est limitée par le phénomène de diffraction qui empêche de focaliser parfaitement les faisceaux lumineux à mieux que $0,5 \mu \mathrm{m}$. Les besoins de l'intégration des composants nécessitent des traits plus fins. Actuellement pour ce travail l'optique est remplacée par des faisceaux d'électrons, des rayons $\mathrm{X}$ ou des faisceaux d'ions.

Les méthodes de la microscopie optique en champ proche devraient permettre de revenir dans certains cas à l'utilisation de la lumière visible. La sonde est utilisée comme une nanosource. Les premières expériences ont utilisé [39-52] des résines photosensibles qui nécessitent un traitement chimique de révélation après l'exposition. La structure ainsi obtenue constitue une réplique de l'intensité lumineuse. Les motifs ainsi tracés sont mesurés à l'aide d'un AFM. Les auteurs ont tracé des traits de $80 \mathrm{~nm}$ de large.

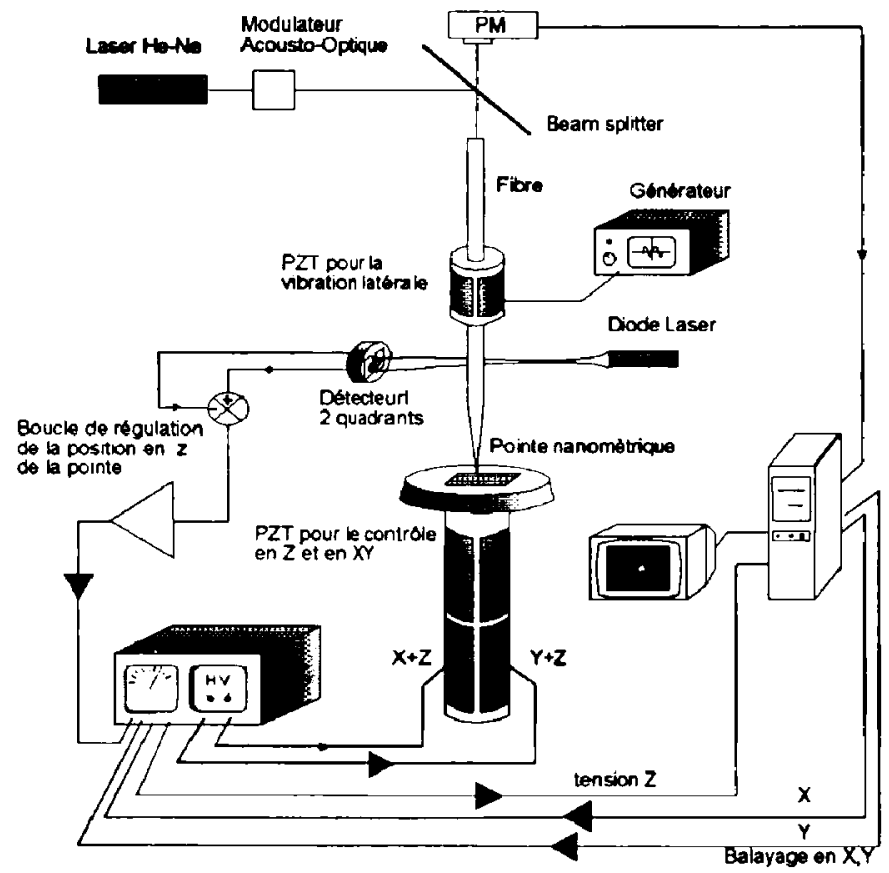

Figure 6 : Schéma d'un microscope par réflexion RSNOM avec asservissement de la position de la pointe par force de cisaillement (Shear-force) [53].

L'utilisation d'un photopolymère, le PMMA-DRI permet d'éliminer l'étape de révélation [53]. Le spot lumineux émis par la pointe crée une déformation mécanique du film de polymère. La forme de l'indentation dépend de la nature de la pointe (pointe métallisée ou pointe diélectrique) et de la dose d'irradiation (intensité lumineuse et durée). Les motifs ainsi photogravés sont relus immédiatement par le même microscope fonctionnant en mesure de force de cisaillement (Shear-force). La figure 6 présente le schéma de principe de ce microscope [53]. La figure 7 présente un exemple de photolithographie, le motif a été tracé et on peut estimer la largeur des traits à $100 \mathrm{~nm}$. 


\subsection{Magnéto-optique et stockage de masse}

Les matériaux magnéto-optiques sont actuellement utilisés pour le stockage de masse de données informatiques. Dans les dispositifs commerciaux actuels, la lecture et l'écriture des données sont effectuées par un spot laser concentré par une lentille. La densité d'information est donc limitée par la diffraction. Avec de la lumière rouge le bit élémentaire a une taille supérieure au micromètre. Quand on pourra utiliser des diodes laser fonctionnant dans le bleu on pourra gagner un facteur deux en dimension ( 4 en intégration).

Les techniques de microscopie optique en champ proche devraient permettre d'augmenter fortement la densité d'intégration.

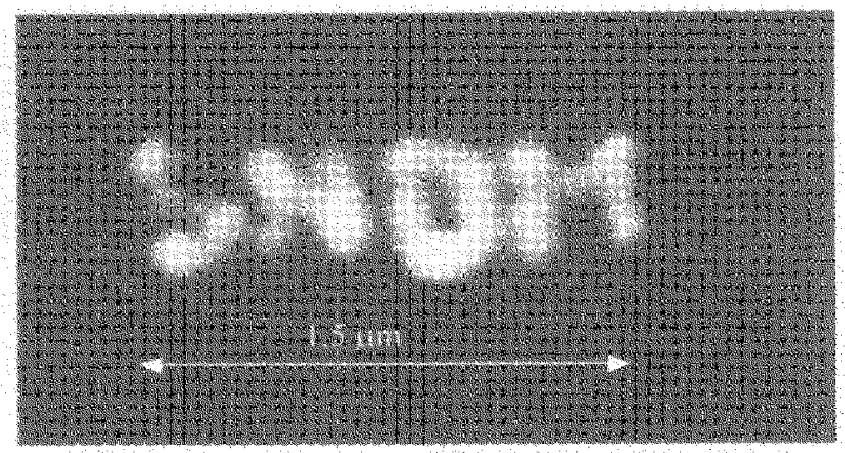

Figure 7 : Exemple de photolithographie sur une résine de PMMA-DR1[53]

Les matériaux sont généralement à aimantation perpendiculaire et ils provoquent une faible rotation du plan de polarisation : effet Faraday en transmission ou effet Kerr polaire en réflexion. Il faut noter que des mesures de polarisation sont plus délicates en champ proche, car la traversée d'une pointe, d'une petite ouverture et d'une fibre optique provoque un changement de polarisation. Ces modifications doivent être connues et corrigées avant toute mesure magnéto-optique.

A l'aide d'un montage STOM, une équipe de l'école polytechnique [54] a réussi à imager des domaines dans des grenats et à observer des détails nanométriques causés par des mouvements de parois. Un montage de SNON avec la pointe en émission a permis à $\mathrm{E}$. Betzig [55] de relire des motifs écrits en champ lointain. De plus la sonde optique peut également être utilisée en écriture. En effet une pointe dont l'extrémité ne fait qu'une dizaine de $\mathrm{nm}$ de diamètre voit se concentrer un très grand éclairement lumineux même si le laser source n'a qu'une puissance de quelques $\mathrm{mW}$. Si la pointe est métallisée, cette extrémité s'échauffe, au delà de $10 \mathrm{~mW}$ de puissance laser la pointe est même détruite. L'échauffement de la pointe peut être suffisant pour porter l'échantilion, situé à quelques nanomètres, au dessus de son point de Curie (environ $200^{\circ} \mathrm{C}$ ). La zone ainsi chauffée perd son aimantation, puis s'aimante en sens opposé en se refroidissant. A l'aide de cette technique un réseau de $20 \mathrm{X} 20$ bits a été inscrit et relu, ce qui porte la densité d'intégration à environ 7 gigabits $/ \mathrm{cm}^{2}$.

D'autres expériences sont en cours pour travailler en réflexion, pour augmenter la densité d'intégration et surtout la vitesse de ces expériences limitées aux environs de $10 \mathrm{kHz}$ ce qui est trop faible pour des applications réelles de stockage de masse. 


\subsection{Physique du solide et matériaux semi-conducteurs}

Dans le domaine des semi-conducteurs il est également obligatoire de disposer de moyens optiques d'observation et d'analyse à l'échelle nanométrique. En simple imagerie signalons l'observation des dislocations dans des films de $\mathrm{Ge}_{x} \mathrm{Si}_{1-x}[56]$. Nous avons signalé qu'une étude spectroscopique en champ proche à la température de l'hélium avait été consacrée à des structures à puits quantique sur de l'AsGa [41]. La mobilité des porteurs dans un film de silicium a été étudiée en champ proche en mesurant la réponse en infrarouge d'une modulation lumineuse dans le visible [56]. Dans des dispositifs à semi-conducteur la sonde optique, utilisée dans le mode d'émission, va créer des porteurs de charge, ce qui va induire un photocourant. Il est donc possible sur de tels objets d'obtenir des images du photocourant en fonction de la position de la sonde optique. Ceci a été effectué sur des lasers à puits quantiques [57]. La figure 8 est une étude de jonction métal semi-conducteur: Au-AsGa. [58]. La jonction est éclairée en champ proche par une sonde métallisée. Le microscope est celui présenté sur la figure 6 . On mesure le photocourant en fonction de la position de la pointe. L'image en "Shear-force » (Fig. 8a) permet de visualiser la structure de l'échantillon, qui est constituée de rubans d'or de $5 \mathrm{~nm}$ d'épaisseur, déposés sur un substrat d'AsGa. L'image de gauche est une inage du photocourant pour une longueur d'onde excitatrice $\lambda=1,33 \mu \mathrm{m}$. Dans cette image, la zone claire qui correspond à un maximum de courant, coïncide avec l'or et l'on constate très peu de diffusion dans le semi-conducteur. Un défaut sur le dépôt d'or est visible. Pour la figure $8 \mathrm{c}$, la longueur d'onde est dans le visible $(\lambda=0,789 \mu \mathrm{m})$ et l'on constate une plus grande diffusion des électrons dans le semi-conducteur. La figure 8 est un excellent exemple de l'association de deux techniques de microscopie à sonde locale pour étudier un même objet.

La microscopie optique en champ proche est également particulièrement adaptée à l'étude des films minces. Une comparaison d'images topographiques, et d'images SNOM en fluorescence de films de Langmuir Blodgett est présentée dans la référence [26]. La sonde d'un microscope tunnel optique utilisée en détection a permis d'observer un plasmon de surface se propageant sur une couche mince d'argent [59].

\subsection{Applications en biologie}

La microscopie électronique par transmission ou à balayage est la technique la plus employée pour obtenir des images d'objets biologiques avec une résolution nanométrique. Mais elle impose de fortes contraintes sur la préparation de l'échantillon : métallisation et observation sous vide. Les microscopies à sonde locale ont apportées de nouveaux moyens d'investigation. Le STM est employé mais nécessite une métallisation préalable. L'AFM est un outil de choix déjà très largement utilisé.

Les premières images d'objets biologiques par un microscope optique en champ proche sont apparues récemment : des images de cellules de tissu cérébral et de cytosquelette de cellule de fibroplaste [38]. Depuis ont été produites des images de virus de la mosaïque du tabac, de bactériophages [60], de chromosomes [49], de flagelles de salmonelles [61]

Ces images ont été obtenues tantôt avec un SNOM ou avec un STOM. Les résolutions ultimes étant voisines de $20 \mathrm{~nm}$.

Dans le domaine des objets biologiques l'AFM et la microscopie optique en champ proche sont des instruments complémentaires. Un microscope mixte AFM-SNOM (ou STOM) semble même être la combinaison idéale pour ce type d'applications. L'AFM apportant les renseignements topographiques et la microscopie optique permettant de conserver en champ proche et en haute résolution toutes les méthodes de marquage par un traceur fluorescent 
largement employées en microscopie optique traditionnelle. D'ailleurs les images biologiques des références [39 et 49] sont des images de fluorescence.
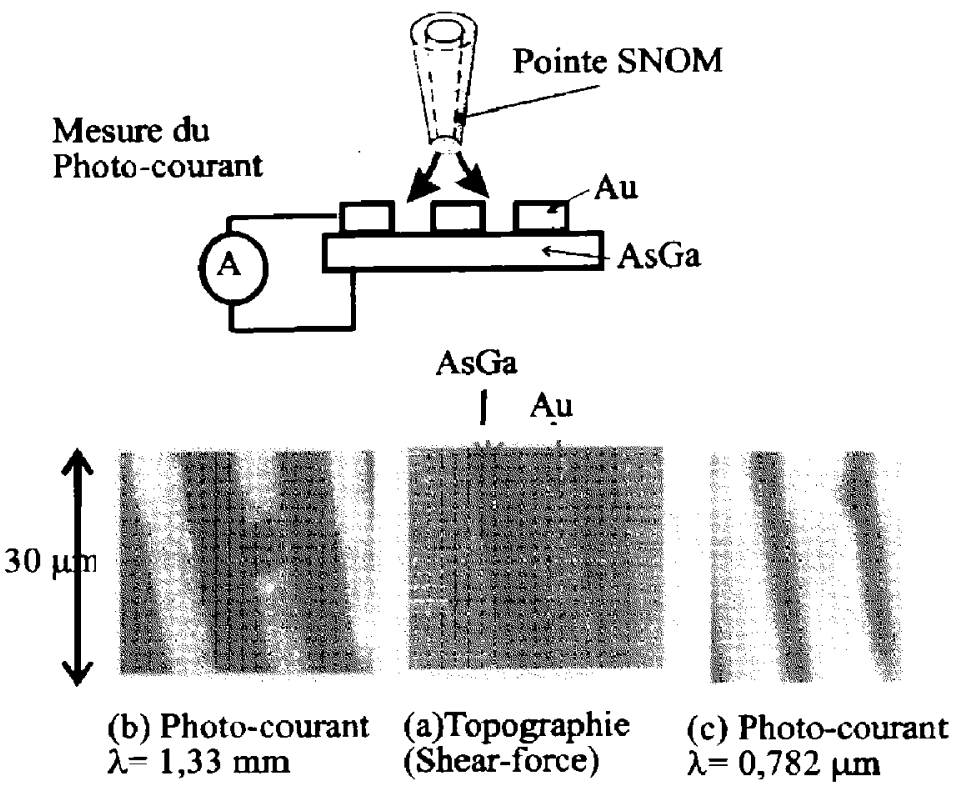

Figure 8 : Utilisation de la microscopie optique en champ proche pour l'étude des jonctions métal-semiconducteur (communication personnelle de S. Davy et M. Spajer)

Des études en biologie moléculaire ont même été commencées. Xie et Dunn ont obtenu des images en fluorescence de protéines [62], de membranes cellulaires intervenant dans la photosynthèse [63]. Ils ont obtenu des images et des spectres de protéines uniques et étudié en champ proche la durée de vie de fluorescence de ces protéines.

\section{CONCLUSION ET PERSPECTIVES}

Dans cet article nous avons présenté rapidement la microscopie optique en champ proche, décrit le principe de fonctionnement, comparé les différents dispositifs, expliqué comment les problèmes techniques étaient résolus. Nous avons montré qu'en simple imagerie, des images en haute résolution montrant des détails d'une dizaine de $\mathrm{nm}$ sont désormais accessibles et que le nm semble bien avoir été atteint. Depuis 2 ou 3 ans les microscopes optiques en champ proche ont dépassé le stade du prototype et de l'appareil de démonstration et le nombre de publications consacrées à de véritables applications se sont multipliées. Nous en avons décrit les principales mais de nombreuses autres sont en train de naître

Du point de vue technique il reste à améliorer la fiabilité et la "convivialité" des appareils existants de façon à les rendre utilisables par des non-spécialistes. Un SNOM est actuellement commercialisé ce qui prouve que ce stade du développement de cette nouvelle microscopie semble être atteint.

Une question reste ouverte, quelle sera la résolution ultime de cette microscopie optique en champ proche ? Contrairement à la microscopie traditionnelle, il ne semble pas exister de 
limite théorique à la résolution en champ proche, mais une limite technologique. L'intensité du signal détecté, le rapport signal sur bruit et surtout la finesse de l'extrémité de la sonde, sont les points essentiels à améliorer si l'on veut augmenter encore la résolution. Sur tous ces points des progrès sont encore à attendre.

Signalons également que des progrès théoriques ont constamment accompagné l'évolution de la microscopie optique en champ proche. L'approche théorique que nous avons employée dans le chapitre (3) était volontairement simplifiée. Elle décrit l'essentiel des phénomènes physiques mais elle est insuffisante pour calculer, même de façon approchée, l'intensité des images des différents microscopes. La référence [64] compare et décrit le principe des différentes approches théoriques permettant ce calcul.

\section{Remerciements}

Je tiens particulièrement à remercier J.M. Vigoureux et Daniel Courjon qui sont à l'origine de mon travail en microscopie optique en champ proche et pour les nombreuses fructueuses discussions scientifiques. Je remercie également les membres du Laboratoire P.M. Duffieux pour leur aide et particulièrement pour les images et dessins, F.Baida, C. Bainier, S. Davy et M.Spajer

\section{Références}

[1] Born M., Wolf E., Principle of Optics (Pergamon Press, 1970).

[2] Roblin G., La microscopie confocale à balayage laser (Techniques de l'ingénieur fiche R6710).

[3] Salvan F., Microscopie par effet tunnel (Techniques de l'ingénieur fiche P895).

[4] Synge E.H.,A suggested method for extending microscopic resolution into the ultramicroscopic region, Phil. Mag., 6 (1928), p. 356-362.

[5] Ash E.A., Nichols G., Super resolution aperture scanning microscope, Nature, 237 (1972), p. 510-512.

[6] Massey G.A., Microscopy and pattern generation with scanned evanescent waves, Apl. Opt., 23 (1984), p. 658-680.

[7] Pohl D.W., Optical near-field scanning microscope, European Patent Application No 0112401 , filed December 27,1982; U.S. Patent 4,604,520, filed December 20 (1983).

[8] Lewis A., Isaacson M., Murray A., Harootunian A., Scanning optical spectral microscopy with 500 A resolution, Biophys. J., 41 (1983), p. 405a.

[9] Pohl D.W., Denk W. et Lanz M., Optical stethoscopy : imaging with $\lambda / 20$, in "Micron and Submicron Integrated circuit metrology," (Ed: K.M. Monahan) Proc. SPIE, 565 (1985), p. 56-61.

[10] Betzig E., Lewis A., Harootunian A, Isaacson V, et Kratschmer E, Near field scanning optical microscopy (NSOM); Development and biophysical applications, Biophys. J., 49 (1986), p. 269-278.

[11] Betzig E., Isaacson $\mathbf{M}$., et Lewis A., Collection mode near-field optical microscopy, Appl. Phys. Lett., 51 (1987), p. 2088-2090.

[12] Reddik R.C., Warmack R.J., Ferrell T.L., New form of scanning optical microscopy, Phys. Rev., B39 (1989), p. 767-770.

[13] Courjon D., Sarayedine K. et Spajer M., Scanning tunneling optical microscopy, Opt. Commun., 71 (1989), p. 23-28. 
[14] de Fornel F., Goudonnet J.P., Salomon L., Lesniewska E., An evanescent field optical microscope, Proc SPIE, 1139 (1989), p. 77-84.

[15] Spajer M., Courjon D., Sarayedine K., Jalocha A., et Vigoureux J.M., Microscopie optique en champ proche par réflexion, Horizons de l'Optique, 30 août 1989, J. Phys III 1 (Paris, 1991), p. 1-12.

[16] Betzig E., Trautman J.K., Harris T.D., Weiner J.S., Kostelak R.L., Breaking the diffraction barrier: optical microscopy on a nanometric scale, Science 251 (1991), p. 1468-1470.

[17] Bainier C., Leblanc S., Courjon D., Scanning tunneling optical microscopy: application to very low relief objects, dans "Near field Optics" édité par D.W. Pohl et D. Courjon ( Kluver Academic, Dordrecht, 1993), NATO Series E 242, p. 97-104.

[18] Specht M., Pedarnig J.D., Heckl W.M., et Hänsch T.W., Scanning Plasmon Near-field microscope, Phys. Rev. Lett., 68 (1992), p. 476-479.

[19] Bachelot R., Gleyzes P.H., et Boccara A.C., Near-field optical microscopy by local perturbation of a diffraction spot., Microsc. Microanal. Microstruct., 5 (1994), p. 389397.

[20] Wickramasinghe H.K., et Williams C.C., Apertureless near field optical microscope, U.S. patent $\mathrm{N}^{\circ} 4947034$ (Août 1990).

[21] Zenhausern F., Martin Y., Wichramasinghe H.K., Scanning interferometric apertureless microscopy: optical imaging at 10 Angstom resolution, Science 269 (1995), pp 1083 1085 .

[22] Vigoureux J.M., Courjon D., Detection of non radiative fields in light of the Heisenberg uncertainty principle and the rayleigh criteriom, Applied Optics, 31 (1992), p. 31703177.

[23] Guerra J.M., Srinivasarao M., Stein R.S., Photon tunneling microscopy of polymeric surface, Science, 262 (1993), p. 1395-1400.

[24] Stamnes J.J., Waves in focal region (Hilger, 1986)

[25] Baida F., Courjon D., Tribillon G., Combination of a fiber and a silicon nitride tip as a bifunctional detector; first results and perspectives, dans "Near field Optics" édité par D.W. Pohl et D. Courjon (Kluver Academic, Dordrecht, 1993), NATO Series E 242, p. 71-78.

[26] Moers M.H.P., Tack R.G., Noordman O.F.J., Segerink F.B., van Hulst N.F., Bölger B., Combined Photon Scanning Tunneling Microscope and Atomic Force Microscope using Silicon Nitride tips, dans "Near field Optics" édité par D.W. Pohl et D. Courjon (Kluver Academic, Dordrecht, 1993), NATO Series E 242, p. 79-85

[27] Betzig E., Finn P.L., Weiner J.S., Combined shear-force and near-field scanning optical microscopy, App. Phys. Lett, 60 (1992), p. 2484-2486.

[28] Ohtsu M., Progress of high-resolution photon scanning tunneling microscopy due to a nanometric fiber probe, Journal of Lightwave Technology, 13 (1995), p. 1200-1210.

[29] Valaskovic G.A., Holton M., et Morisson G.H., Parameter control, characterization, and optimization in the fabrication of optical fiber near-field probe, Applied Optics, 34 (1995), p. 1215-1228.

[31] Fisher U.C., Zapletal M., The concept of a coaxial tip as a probe for scanning near field optical microscopy and steps toward a realisation, Ultramicroscopy, 42-44 (1992), p. 393-398.

[32] Fisher U.C., The tetrahedral tip as a probe for scanning near-field optical microscopy dans "Near field Optics" édité par D.W. Pohl et D. Courjon (Kluver Academic, Dordrecht, 1993), NATO Series E 242 p. 255-262. 
[33] Danzebrink H.U., Fischer U.C., The concept of an optoelectronic probe for near field microscopy, dans "Near field Optics" édité par D.W. Pohl et D. Courjon (Kluver Academic, Dordrecht, 1993), NATO Series E 242 p. 303-308.

[34] Kolb G., Karrai K., Abstreiter G., Optical near-field induced current microscopy, Appl. Phys. Lett., 65 (1994), p. 3090-3092.

[35] Betzig E., Krubb S.G., Chichester R.J., DiGiovanni D.J., Wiener J.S., Fiber laser probe for near-field scanning optical microscopy, Appl. Phys. Lett., 63 (1993), p. 3550.

[36] Kopelman R., Tan W., Shi Z.Y., Bimhaum D., Near-field optical and exciton imaging. Spectroscopy and chemical sensor, dans "Near field Optics" édité par D.W. Pohl et D. Courjon (Kluver Academic, Dordrecht, 1993), NATO Series E 242, p. 17-24.

[37] Courjon D., Baida F., Bielefeld H., Spajer M., New compact stand alone optical nearfield/AFM microscope, Proceedings of Mecatronics, Besançon, 1996.

[38] Boccara C.A., Voir plus petit, Pour la Science, 227 (1996), p. 30.

[39] Betzig E., Principles and applications of near-field scanning optical microscopy (NSOM) dans "Near field Optics" édité par D.W. Pohl et D. Courjon (Kluver Academic, Dordrecht, 1993), NATO Series E 242, p. 7-15.

[39] Betzig E., J.K. Trautman, Near-field optics: microscopy, spectroscopy, and surface modifications beyond the diffraction limit, Science, 257 (1992),p. 189-195

[40] Grober R.D., Harris T. D., Trautman J.K., Betzig E., Design and implementation of a low temperature near-field scanning optical microscope, Rev. Sci Instrum., 65 (1994), p. 626-631.

[41] Grober R. D., Harris T. D., Trautman J.K., Betzig E., Wegsheider W., Pfeiffer L., West K., Optical spectroscopy of a GaAs/AlGaAs quantum wire structure using near-field scanning optical microscopy, Appl. Phys. Lett., 64 (1994),p. 1421-1423.

[42] Betzig E., Chichester R. J., Single molecules observed by near-field scanning optical microscopy, Science, 262 (1993),p. 1422-1425.

[43] Moerner W.E., Plakhonik T., Imgartinger T., Wild U. P., Near-field optical spectroscopy of individual molecules in solids, Phys. Rev. Lett, 73 (1994), p. 27642767.

[44] Trautman J.K., Macklin J.J., Brus L.E., Betzig E., Near-field spectroscopy of single molecule at room temperature, Nature, 369 (1994), p. 40-42

[45] Pedarnig J.D., Specht M., Hänsch T.W., Fluorescence lifetime variations and local spectroscopy in scanning near-field optical microscopy, dans "Photon and Local probes" édité par O. Marti et R. Möller (Kluver, Dordrecht, 1995), Nato Series E 300, pp 151163.

[46] Xie X.S., Dunn R.C., Probing single molecule dynamics, Science, 265 (1994), p. 46

[47] Ambrose W.P., Goodwin P.M., Martin P.M., Keller R.A., Alteration of single molecule fluorescence lifetimes in near-field optical microscopy, Science, 265 (1994), p. 364-367.

[48] Piednoir A., Creuzet F., Licoppe F., and Ortéga J.M., Locally resolved infrared spectroscopy, Ultramicroscopy, 57 (1995), p. 282-286.

[49] van Hulst N., Moers M., Borgonjen E., Applications of near-field optical microscopy : fluorescence in situ hybridisation, Langmuir-Blodgett films and integrated optical waveguides, dans Photon and Local Probe, édité by O. Marti and R. Möller (1995), NATO ASI Series E, 300 pp 165-187.

[50] Buratto S.K., Hsu J.W.P., Trautman J.K., Betzig E., Bylsma R.B., Bahr C.C., Cardillo M.J., Imaging InGaAsP quantum-well lasers using near-field scanning optical microscopy, J. Appl. Phys., 76 (1994), p. 7720.

[51] Hörsch 1., Kusche R., Marti O., Weigl B., Ebeling K.J., Spectrally resolved near-field imaging of vertical cavity semiconductor lasers, J. appl. Phys., 79 (1996), p. 3831-3834. 
[52] Krausch G., Wegscheider S , Kirsch A., Bielefeld H., Meinerrs J.C., Mlynek J., Nearfield microscopy and lithography with uncoated fiber tips: a comparison, Opt. Comm, 119 (1995), p. 283-288.

[53] Davy S., Rachard G., and Spajer M., Lithography on PMMA-DR I with reflection near field optical microscopy (R-SNOM) and probe chracterization, SPIE, 2782 (1996), p. 551-558.

[54] Safarof V.1., Kosobukin V.A., Hermann C., Lampel G., Marlière C., Peretti J., Nearfield magneto-optics with polarization sensitive STOM, Ultramicroscopy, 57 (1995), p. 270-277.

[55] Betzig E., Trautman J.K., Wolfe R., Gyorgy E.M., Finn P.L., Kryder M.H., Chang C.H., Near-field magneto-optics and high density data storage, Appl. Phys. Lett, 61 (1992), p. 142-144

[55] Hsu J.W.P., Fitzgerald E.A., Xie Y.H., Silverman P.J., Near-field scanning optical microscopy imaging of individual threading dislocations on relaxed $\mathrm{Ge}_{\mathbf{x}} \mathrm{Si}_{1-\mathrm{x}}$ films, App. Phys. Lett, 65 (1994), p. 344-346.

[56] LaRosa A., Jahncke C.L., Hallen H.D., Time as a contrast mechanism in near-field imaging, Ultramicroscopy, 57 (1995), p. 303-308.

[57] Buratto S.K., Hsu J.W.P., Trautman J.K., Betzig E., Bylsma R.B., Bahr C.C., Cardillo M.J., Near-field photoconductivity. Application to carrier transport in InGaAsP quantum well lasers, Appl. Phys. Lett., 65 (1994), p. 2654-2656.

[58] Almeda J,, dell'Orto T., Coluzza C., Margaritondo G., Novel spectrometry: Pt-Gap studies by spatially resolved photoemission with near-field optics, App. Phys. Lett., 69 (1996), p. 2361-2363.

[59] Dawson P., Smith K.W., de Fornel F., Goudonnet J.P., Imaging of surface plasmon launch and propagation using a photon scanning tunneling microscope, Ultramicroscopy, 57 (1995), p. 287-293.

[60] Jiang S., Ohsawa H., Yamada K., Ikai A., Nanometric scale biosample observation using a photon scanning tunneling microscope, Jap. J. Appl. Phys., 31 (1992), p. 2282-2287.

[61] Naya M., Mononobe S., Maheswari R. U., Saiki T., Ohtsu M., Imaging of biological samples by a collection-mode photon scanning tunneling microscope with an apertured probe, Opt. Comm., 124 (1996), p. 9-15.

[62] Dunn R.C., Allen E.V., Joyce S.A., Anderson G.A., Xie X.S., Near-field imaging of single proteins, Ultramicroscopy, 57 (1995), p. 113-117.

[63] Dunn R.C., Holton G.R., Metz L., Xie X.S., J. Phys. Chem, 98 (1994), p. 3094-3098.

[64] Van Labeke D., Barchiesi D., Theoretical problems in scanning near-field optical microscopy, édite par D.W. Pohl et D. Courjon (Kluver Academic, Dordrecht, 1993), NATO Series E 242, pp 157-178. 